\title{
OWL instrument concept studies
}

\author{
G. Monnet \& S. D'Odorico \\ European Southern Observatory, Karl-Schwarzschild-Strasse 2, \\ D-85748 Garching, Germany \\ email: gmonnet@eso.org
}

\begin{abstract}
The 8-instrument suite studied by the ESO community in the frame of the conceptual study of the $100 \mathrm{~m}$ OWL telescope is briefly presented. Potential capability for unique science and the main technical challenges are identified.
\end{abstract}

Keywords. instrumentation — telescopes.

\section{Introduction}

Since almost 10 years, ESO has pursued the concept study for a giant optical-infrared telescope with a primary mirror diameter up to 100 meters. Dubbed OWL for the eponymous bird keen night vision, it relies in particular on serialized production of identical spherical segments for the primary mirror (flat for the secondary) and of a very small number of standard basic elements for its fractal-like mechanical structure.

One essential component of this effort was to derive a suite of instruments that could both support the most important science cases identified for the facility and cover enough of potential observing parameters to ensure a large discovery space when it is in full operation 10-15 years from now. This paper presents a) the scope and setting-up of these instrument studies, b) a short summary for each of the 8 instrument concepts selected and c) overall results and future actions.

The OWL concept study has undergone a comprehensive Review by an International Board on November 2-5, 2005. This is expected to lead to the full definition of a European Extremely Large Telescope (ELT) project by the end of 2006 (see "Status of the ESO ELT", G. Monnet, this Workshop), followed by design and construction of the facility.

\section{OWL instrument concept studies - results}

Eight, roughly 9-month long, studies were launched in the fall of 2004. At this time, an extensive science case for a 50-100 m ELT had been thoroughly explored by a large segment of the European astrophysical community under the aegis of the European Commission sponsored OPTICON program (G. Gilmore, Cambridge University, Coordinator). Furthermore, a detailed outline of OWL's basic opto-mechanics was by then available, plus a very first iteration of the many Adaptive Optics (AO) systems critically required for any ELT. The instrument suite retained for the studies has been largely derived from the most important science case needs, viz. identify the $1^{\text {st }}$ light in the Universe, unravel the basic steps in the development of its structures, identify individual stars in external galaxies and, finally, detect and characterize exo-planets, possibly down to Earth-like ones.

Basic objectives for the instrument concept studies were to support the science cases with feasible and affordable instrument concepts, check the interfaces between the instruments on the one hand and the telescope with its AO systems on the other hand and 


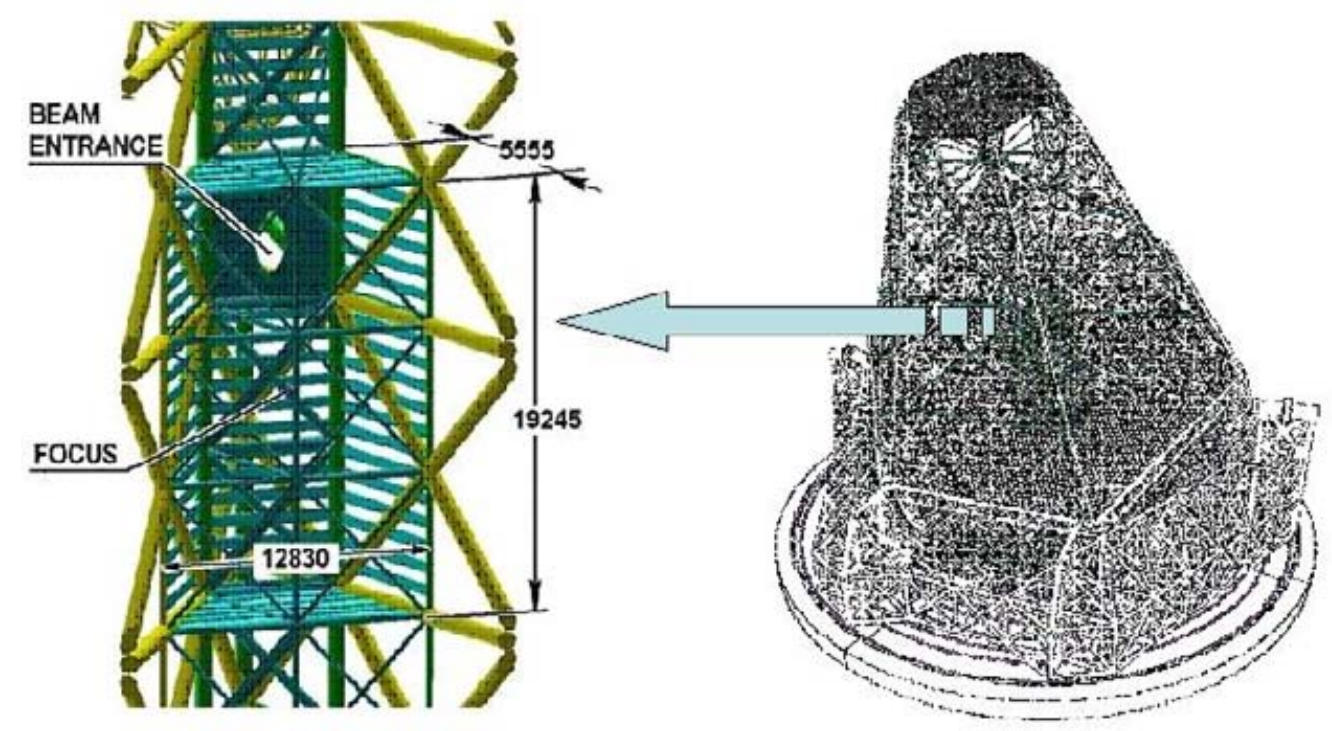

\section{1 of 6 Instrument "rooms"}

Figure 1. OWL (right) and one of its six instrument rooms (left).

identify the basic enabling technologies required to develop the instruments. It should be noted that this constitutes only the first round in an iterative process, with most of the feedback to the telescope design to be provided in 2006. Note also that this instrument suite does not pretend to cover all important science which could be envisioned with OWL.

All in all, more than 150 scientists and engineers in seven European countries participated to the effort. Choices for the host institutes and team members were based on both competence and availability at short notice, with six studies led by external PI and two by ESO PIs. The studies were steered by Statements of Works. They were supported by the OWL telescope interface document, an exposure time calculator, feedback from the ESO telescope design and adaptive optics groups and by an ESO instrument scientist attached to each project. Figure 1 shows the basic telescope layout (right) and one of its six instrument "rooms" (left). Designing instruments for OWL turned out to be a tough call with hard AO-related requirements, gravity-variant foci only and an F/6 input beam with a very small back focal distance.

The study teams were asked to a) develop further the high priority science cases to get detailed instrument requirements, b) derive an instrument concept and estimate its performance, volume, mass and cost, c) assess instrument performance in the context of present and future key facilities (e.g. JWST, ALMA) for a 50-100 m telescope diameter range, d) identify critical instrument components and subsystems and e) identify special requirements directed to the telescope design, including its $\mathrm{AO}$ capabilities.

The eight studies were presented and discussed at ESO end of September 2005. They include detailed quantitative science cases and advanced instrument concepts. All final reports were delivered by October 20, 2005. They represent a total of 1200 A4 pages and are available on a DVD (enquiries should be addressed to sdodoric@eso.org). 


\begin{tabular}{|c|c|c|c|}
\hline PROJECT & $\lambda(\mu \mathrm{m})$ & GOALS & DESCRIPTION \\
\hline CODEX & $0.4-0.7$ & $\begin{array}{c}\text { Universe } \\
\text { Deceleration }\end{array}$ & $\begin{array}{l}\text { High-accuracy radial } \\
\text { velocity Spectrometer }\end{array}$ \\
\hline QuantEYE & $0.4-0.8$ & $\begin{array}{l}\text { Extreme physical } \\
\text { environments }\end{array}$ & Ultra-fast Spectro-Imager \\
\hline HyTNIC & 1.1-1.6 & Planetary Disks & $\begin{array}{c}\text { High Contrast } \\
\text { diffraction-limited Imager }\end{array}$ \\
\hline EPICS & $0.6-1.9$ & Exo-planets & $\begin{array}{c}\text { Extreme Contrast } \\
\text { Spectro-Imager }\end{array}$ \\
\hline MOMFIS & $0.8-2.5$ & High-z galaxies & $\begin{array}{l}\text { Multi-IFU Spectrometer } \\
\text { in large patrol field }\end{array}$ \\
\hline ONIRICA & $0.8-2.5$ & $\begin{array}{l}\text { Stellar populations } \\
\& \text { high-z finder }\end{array}$ & $\begin{array}{c}\text { NIR Wide-Field } \\
\text { diffraction-limited Imager }\end{array}$ \\
\hline T-OWL & $2.5-20$ & $\begin{array}{l}\text { Planets \& Stellar } \\
\text { formation at all z }\end{array}$ & $\begin{array}{c}\text { Thermal IR } \\
\text { Spectro-Imager }\end{array}$ \\
\hline SCOWL & $\begin{array}{c}250- \\
450-850\end{array}$ & Dusty Universe & $\begin{array}{l}\text { Wide-Field sub-mm } \\
\text { Imager }\end{array}$ \\
\hline
\end{tabular}

Table 1. OWL Instrument Suite

\section{Instrument Concepts}

Table 1 summarizes the eight instrument conceptual studies (CODEX, QuantEYE, HyTNIC, EPICS, ONIRICA, MOMFIS, T-OWL and SCOWL) while Figure 2 shows a bird's view of the whole set.

\section{Instrument Concepts}

\subsection{CODEX}

The COsmic Dynamics EXperiment (CODEX) concept was developed by 22 staff from 4 institutes (ESO, IoA-Cambridge, Observatoire de Genève and INAF-Trieste). Its PI is L. Pasquini (ESO), with S. Cristiani and P. Molaro (Trieste), M. Haehnelt (IoA) and F. Pepe (Genève) as co-PIs. Detailed presentations of the project can be found in Pasquini et al. (2006) and Molaro et al. (2006).

CODEX primary science goal is to test our universe cosmological model from measures of over at least a period of 10 years of Lyman- $\alpha$ forest and metal systems radial velocity shifts in the direction of distant QSOs due to cosmic acceleration. Additional science goals are the detection of an eventual cosmological variation of the fine structure constant, the search for extra-solar terrestrial planets via their radial velocity signatures and the measure of primordial ${ }^{7} \mathrm{Li}$ abundance and ${ }^{6} \mathrm{Li} /{ }^{7} \mathrm{Li}$ ratio.

The instrument concept features six high-resolution $(\sim 200000)$ identical spectrometers in order to fully use the photons coming from a point-like object (seeing-limited) and collected by a $100 \mathrm{~m}$ telescope. It covers the 400 to $800 \mathrm{~nm}$ wavelength range. A highly demanding $1 \mathrm{~cm} / \mathrm{s}(1 \sigma)$ stability over at least 10 years of repeated observing must be achieved to reach the prime science goal. This is a tough challenge which requires in 


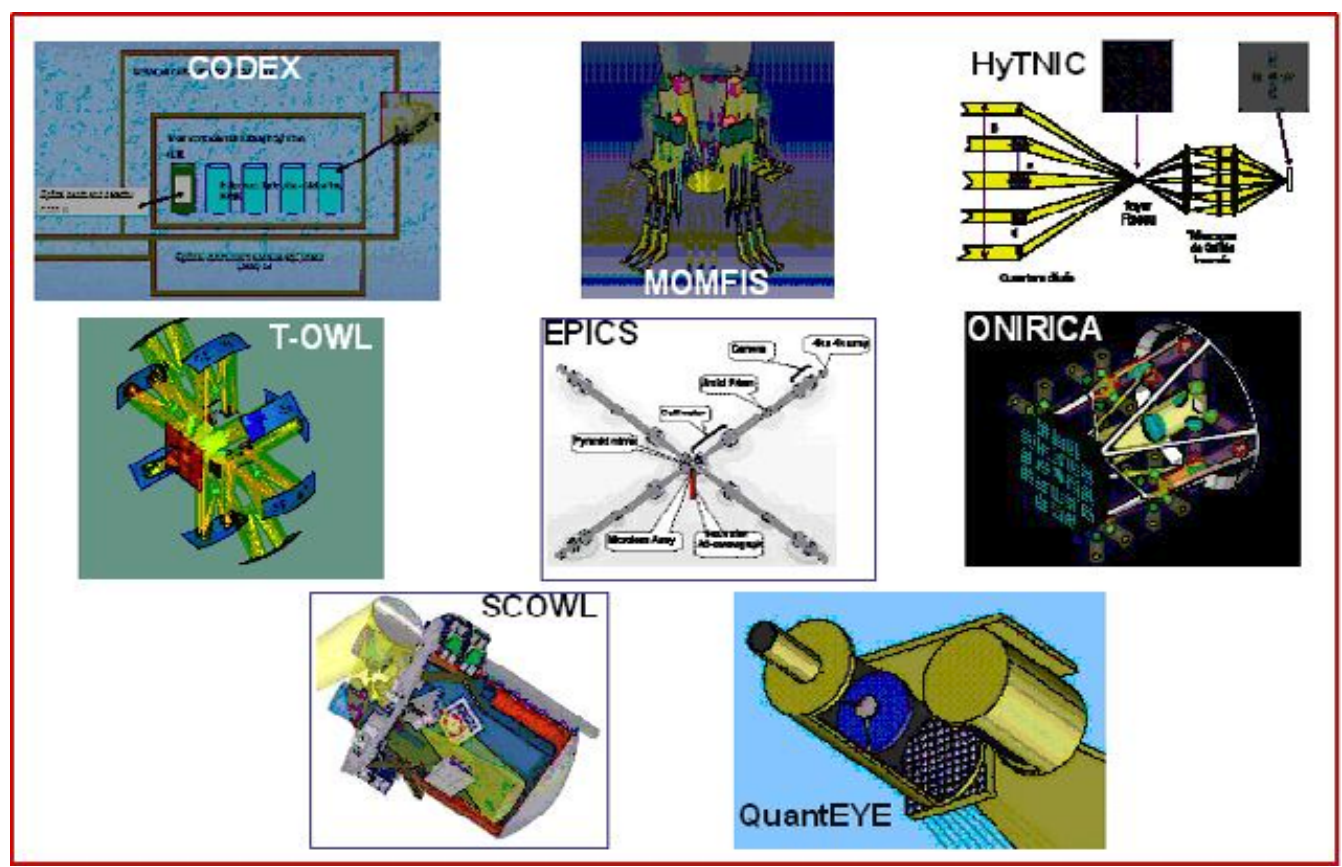

Figure 2. A bird's view of the eight instrument concepts

particular a large gravity invariant, thermally stable (a few $\mathrm{mK}$ ) laboratory fed by a coudé train, an efficient fiber-based light scrambling system and an absolute metrological reference, probably provided by a laser comb system.

This approach can easily be scaled down for a smaller size ELT, with e.g. only 1 spectrometer needed for a $40 \mathrm{~m}$ telescope. However, the observing time required to achieve the prime science goal might have to extend well over ten years.

\subsection{QuantEYE}

The Quantum Optics Instrumentation for Astronomy (QuantEYE) concept was developed by 11 staff from 5 institutes (Lund Observatory, Department of Engineering-Padova University, ST-ECF/ESO and INAF-Padova Observatory). Its co-PIs are D. Dravins (Lund) and C. Barbieri (Padova). A detailed description can be found in Dravins et al. (2006) and Barbieri et al. (2006).

The QuantEYE approach is to explore for the first time photon arrival statistics of astrophysical targets at time resolutions much smaller than 1ms and possibly down to 10ns. It will constitute a new kind of probe for extreme physical environments like millisecond pulsars, variability near black holes, white dwarf surface convection, oscillations and surface structures of neutron stars, photon bubbles inside accretion flows, free-electron lasers around magnetars and natural laser-line emissions.

This translates into a small size, relatively simple (no AO correction required) doublehead ( 1 object plus 1 reference star), very fast photometer working in the visual-red range. Sensitivity gain with increasing telescope size is extremely steep, going as the $4^{\text {th }}$ power of diameter for $2^{\text {nd }}$ order photon correlation and even with the $8^{\text {th }}$ power for $4^{\text {th }}$ order correlation. An ELT of any size thus promises big gains over what could be presently achieved with $8-10 \mathrm{~m}$ telescopes. The main challenge for QuantEYE is to develop relatively small $(10 \times 10$ for a $100 \mathrm{~m})$ but extremely fast diode arrays, handle 
the large data flow and, especially, separate astrophysical signals from atmospheric and internal (e.g. telescope vibrations) spurious effects.

\section{3. $H y T N I C$}

The Hyper-Telescope Near-IR Camera (HyTNIC) concept was developed by the LISECollège de France Laboratory (O. Lardière, PI). Initial impetus for this study came from the strong wish to be able to use the $100 \mathrm{~m}$ OWL for astrophysical observations in the 5-year period during which the primary mirror area is being filled from an initially relatively meager set of 254 segments to its full 3048 complement, while the facility can already be operated.

The concept is based on Labeyrie's Hyper-Telescope approach in which a sparsely filled pupil is densified, using inverted Galilean telescopes put on each sub-pupil to relay the initial "Fizeau" focus to the final focus with a significant gain in energy concentration. Main science goal of the system is direct detection by speckle interferometry of hot Jupiters 10,000 times fainter than their parent stars. Extensive simulations have been made to maximize the ability to detect faint companions, while respecting the strong technical constraint that all mirror segments must be laid in a simply connected format to permit global phasing of the primary mirror at anytime. The optimum strategy found is to lay down 775 segments in a "spider-web" diluted format during the first two years, to be completed by a growing compact array during the following 3 years. The diluted part offers the diffraction limit of a $100 \mathrm{~m}$, while the compact ones provides for the equally required high dynamics.

\subsection{EPICS}

The Exo-Planet Imaging Camera Spectrograph (EPICS) concept study was coordinated by ESO (N. Hubin, M. Kasper and C. Vérinaud) and developed by 43 staff from 14 institutes (INAF-Padova, ETH-Zürich, AI-University of Amsterdam, LAOMP-Toulouse, ENS-Lyon, MPIA-Heidelberg, LAOG-Grenoble, ONERA-Chatillon, UKATC-Edinburgh, LAM-Marseille, LESIA-Observatoire de Paris, American Museum of Natural HistoryNew York, ASTRON-Groningen and MPS-Lindau). The instrument concept is presented in detail by Beuzit et al. (2006).

EPICS addresses OWL's critical science case of direct detection and characterization of exo-planets. Its primary goal is to study rocky planets in habitable zones from $\mathrm{H}_{2} \mathrm{O}, \mathrm{CO}_{2}$ and $\mathrm{O}_{2}$ signatures; a secondary one is to study evolved giant gaseous planets from $\mathrm{CH}_{4}$. It faces the huge technical challenge to get very high Strehl images at down to reddish wavelengths, although over a very small field graced with a bright central "guide" star, and reach extremely high contrast values, up to 10 billion to detect an exact simile of our Earth.

This requires a highly elaborate eXtreme-AO (XAO) system with a coronagraphic facility and, equally important, innovative post-corrected focus instruments and sophisticated observing strategies, providing extremely well calibrated differential data to boost the contrast. The project features 3 differential instruments, viz. an imager, a polarimeter and an integral field spectrometer. Such a "Planet Finder" is currently under development for one of the VLT $8 \mathrm{~m}$ Unit Telescope and considered as a pathfinder for EPICS.

The EPICS concept is essentially telescope-size invariant, but its scientific goals are not, with potential contrast scaling sharply as the $4^{\text {th }}$ power of telescope diameter albeit only if similar Strehl ratios are achieved independently of telescope size. Jupiterlike exo-planets might thus be barely observable with an 8-10 m, but should be easily within reach with $25-50 \mathrm{~m}$ ELTs, while a few Earth-like planets might (but also might not) be accessible with an 80-100 m ELT. 


\subsection{ONIRICA}

The OWL NIR Imaging CAmera (ONIRICA) concept study has been developed by a staff of 14 from 5 institutes (INAF-Arcetri, INAF-Roma, INAF-Bologna, INAF-Padova and MPIA- Heidelberg). Its PI is R. Ragazzoni (Arcetri).

ONIRICA is conceived as a workhorse near-IR diffraction-limited imager for OWL. Its basic objective is to fully exploit OWL's large light collection and concentration advantage for observations of stellar objects over smaller space (e.g. JWST) and groundbased telescopes. The primary science goal explored by the ONIRICA team was the construction of the color-magnitude diagrams in galaxies to get their star formation history over a large redshift range. Of particular importance is the ability to measure individual stars in elliptical galaxies in the Virgo clusters.

The result is a "superb seeing" $J-H-K$ imager, using Multi-Conjugate AO (MCAO) correction to get $\sim 30 \%$ Strehl in the central $1^{\prime}$ diameter field with a very good sky coverage, using natural guide stars only. The concept also features an outer $5^{\prime}$ diameter field sampled at $\sim 20$ mas, with improved seeing images provided by a Ground-Layer AO (GLAO) system. This concept faces significant challenges, namely building an efficient MCAO system and a very large cryogenic F/1.5 Instrument, and deploying a huge (close to 1 billion pixels) and likely expensive near-IR scientific detector array. Equally important will be to develop optimized data extraction algorithms to derive accurate stellar magnitudes from images which point-spread function varies very substantially in time, over the field and, especially, with wavelength. Note also that the wide-field mode would greatly benefit from a good level of $\mathrm{OH}$ suppression, e.g. given by custom-made interference filters.

\subsection{MOMFIS}

The Multi Object Multi Field Infrared Spectrograph (MOMFIS) concept has been developed by a staff of 18 from 5 institutes (LAM-Marseille, GEPI and LESIA-Observatoire de Paris, CRAL-Lyon and ONERA-Chatillon). Its PI is J.G. Cuby (LAM) who is presenting the concept in detail in Cuby et al. (2006). The critical, innovative MOAO technique (see below) is presented by Neichel et al. (2006).

The MOMFIS prime science goal is the spectroscopic study of the first galaxies in the Universe $(z \sim 7-10)$ to get their luminosity function, mass assembly and star formation history as well as the inter-galactic medium metal content and the epoch of reionization. Targets could be obtained from other ELTs, from the ONIRICA OWL near-IR camera and from JWST. To get a reasonable observing efficiency, up to 30 object pickers each feeding a science object to a medium format $(\sim 30 \times 30$ pixels $)$ Integral Field Unitpatrolling a large $\left(\sim 3^{\prime} \times 3^{\prime}\right)$ field are needed.

The concept relies on a 2-stage AO correction, a Ground-Layer AO (GLAO) system provided by the telescope itself, followed by a Multi-Object Adaptive Optics (MOAO) system with AO "guide star" buttons put on the brightest stars in the field and AO "science" buttons put on the astrophysical targets. Every AO button gets its own mini deformable mirror, with the "guide star" ones working in classical closed-loop with their own individual wavefront sensors, while the "science" ones are directly driven in pseudoclosed loop by the overall system real-time computer.

The "science" buttons then feed an array of 30 identical near-IR cryogenic spectrometers, providing either $J, H$ or $K$ spectra in one shot at $\sim 4000$ spectral resolution. Using natural guide stars only, $30 \%$ encircled energy can be expected in the $K$-band under median seeing condition and over much of the sky. Besides relying on a new - and demanding - AO technique, we are dealing here with a complex, large, massive instrument 
with a large number of moving devices requiring high - if "only" $8 \mathrm{~m}$ type - stability during exposures.

\section{7. $T-O W L$}

The thermal infrared imager and spectrograph for OWL (T-OWL) concept has been developed by a staff of 26 from 4 institutes (MPIA-Heidelberg, Leiden University, ASTRONGroningen and ESO). Its PI is R. Lenzen (MPIA) and co-PI B. Brandl (Leiden). The T-OWL concept is presented in detail by Lenzen et al. (2006).

The effort was aimed at designing a spectro-imager in the thermal domain (3 to $27 \mu \mathrm{m}$ ) which could deliver much better spatial resolution than JWST and advantages in high resolution spectroscopic. With sub-10 mas spatial resolution over the whole wavelength range and spectroscopic resolution up to 50000, the concept addresses a large variety of astrophysical targets, from solar system objects to high-redshift active galactic nuclei. With the large detectivity gains envisioned, a number of scientific studies not presently pursued in the thermal domain, like Galactic pulsars and high-redshift gamma-ray bursts, will actually become accessible on ELTs. One particularly promising and exciting domain is the study of exo-solar planets and proto-planetary systems. Direct detection of exoEarths through high-resolution spectrometry in the $N$-band might even become possible, but this crucially depends on getting at least OWL's full $100 \mathrm{~m}$ size.

T-OWL could be seen as an (almost) first light OWL instrument. It is relatively light and needs only the moderate GLAO correction provided by the telescope itself with natural guide stars only. One delicate issue, however, is the need for a "thermally-cleaned" telescope, including possibly a fast chopping capability, a daring requirement for any ELT and particularly for a $100 \mathrm{~m}$ diameter one. Optimizing for the whole thermal domain also impacts significantly on site requirements, in particular for the $L$-band which would gain most from a very dry location.

\subsection{SCOWL}

The Submillimeter Common User Bolometer Array for OWL (SCOWL) concept study has been developed by a staff of 7 from 2 institutes (UKATC-Edinburgh and ESO). Its $\mathrm{PI}$ is B. Dent (UKATC).

It is conceived as a survey facility for deep, large-scale mapping of dust-absorbed stellar formation regions. It will in particular be able to probe the role of star-forming regions in the early universe, down to a few $\mathrm{L}^{\star}$ galaxy even at very high-redshift. The instrument concept provides $2^{\prime} .5$ imaging in 3 simultaneous sub-mm bands $(350,450,850 \mu \mathrm{m})$ at $1^{\prime \prime}$ spatial resolution with down to $0.1 \mathrm{mJy}$ sensitivity. It offers a much faster survey capability than the ALMA Interferometric Array, being actually an ideal complement in selecting promising targets to be later characterized spectroscopically by ALMA.

With no AO requirements and a relatively "simple" instrument - albeit with a large cryogenic vessel hosting a huge bolometer array - SCOWL is seen as a first light OWL instrument. It should be noted that its capabilities depend very much on being located in an extremely dry site, especially for the highest frequency bands.

\section{Overall results and future actions}

This very significant effort in a short time span has already unraveled a whole suite of instrument concepts, able to address the "overwhelming" science cases expected to be accessible with an up to $100 \mathrm{~m}$ ELT. These are systems mainly based on proven technology, relying on the basic blocks developed for the present generation of 8-10 m telescopes, but rearranged in new "phyla", as can be glanced from Figure 2. 
Required performance appears within technical reach even for a telescope as large as $100 \mathrm{~m}$ with a big proviso however: for most of the applications the operation of the complementary AO system is relying on natural guide stars only. This might result in incomplete sky coverage and even more so if the diameter of the telescope should be substantially smaller than $100 \mathrm{~m}$. The addition of a laser guide star facility will have to be studied.

Much R\&D effort is still needed in a number of instrumental and Adaptive Optics critical areas, partly to ensure technical feasibility per se, but also to design actual instruments near the end of the present decade in a more cost-performance effective way that we would be able to do if starting right now. With the cost of a major OWL instrument expected to be in the 25 MEuros (capital) plus 250 person-years range, this is a particularly important angle to follow. From the above, a by far not all inclusive list could feature both large and small deformable mirrors with high actuator numbers, MOAO and possibly other new AO concepts (including versions based on laser guide stars), all associated AO paraphernalia, affordable high-quality infrared arrays, simple but accurate metrological systems, reliable high-performance cryogenic motors, cryogenic image slicers, etc. For much more detailed presentations, see the papers by Cunningham et al. (2006)(Novel Technologies for ELT Instruments), Hubin et al. (2006)(AO for ELTs) and Hodapp (2006)(Introduction to Detectors).

In Europe a large technological effort has started under the EC-sponsored ELT Design Study (see the ELT-DS paper by Ardeberg et al. (2006)). It is aimed at developing generic technologies critically required for ELTs, with particular emphasis on AO-related areas. On the instrumental side, the concepts presented here are being further explored within the ELT-DS Study (responsible, C. Cunningham). Seven so-called "small" studies have started in September 2005; they will be followed by at least 3 Point Design Studies to be selected in a year. Feedback to and from the ESO ELT Telescope Design effort is essential and will be actively pursued.

Equally vigorous ELT-related technological programs are underway on the NorthAmerican continent and elsewhere. Increasing cooperation level between these various efforts is crucial to ensure that the challenging development of the new generation of Extremely Large Telescope is a success.

\section{Acknowledgements}

The authors want to thank warmly the eight instrument teams for their in-depth essential contribution to the definition of the future European ELT.

\section{References}

Ardeberg, A., Andersen, T. \& Owner-Petersen, M. 2006, these proceedings

Barbieri, C. et al. 2006, these proceedings

Beuzit, J.-L. et al. 2006, these proceedings

Cuby, J.-G. et al. 2006, these proceedings

Cunningham, C. et al. 2006, these proceedings

Dravins, D. et al. 2006, these proceedings

Hodapp, K. 2006, these proceedings

Hubin, N. et al. 2006, these proceedings

Lenzen, R., Brandl, B. \& Brandner, W. 2006, these proceedings

Molaro, P. et al. 2006, these proceedings

Neichel, B. et al. 2006, these proceedings

Pasquini, L. et al. 2006, these proceedings 